\title{
Hinweis zur Zitierweise und zur Nomenklatur
}

Veröffentlichte inländische Gerichtsentscheidungen sind, soweit in der entsprechenden autorisierten Entscheidungssammlung (insbesondere BGHZ, BPatGE, RGZ) veröffentlicht, nach dieser zitiert, weiter und sonst nach einer Zeitschriftenfundstelle (grundsätzlich in der Reihenfolge GRUR, GRUR Int, Mitt, InstGE, BlPMZ, NJW und GRUR-RR, sonstige), Entscheidungen des BGH und des RG auf dem Gebiet des gewerblichen Rechtsschutzes und benachbarten Gebieten zusätzlich mit einem Schlagwort. Nicht oder nur im Leitoder Orientierungssatz im Druck veröffentlichte Entscheidungen sind regelmäßig mit Entscheidungsdatum, Aktenzeichen und Fundstelle des Leit- oder Orientierungssatzes mit dem Zusatz „Ls“ oder „KT“ (für Kurztext) zitiert. Soweit im Einzelfall undokumentierte Entscheidungen des BPatG berücksichtigt sind, ist dies im allgemeinen erwähnt („undok“). Entscheidungen sonstiger Instanzgerichte ohne Veröffentlichungsangaben sind vielfach undokumentiert, teilweise aber im Internet verfügbar. Entsprechendes gilt für Entscheidungen anderer inländischer Stellen (insbesondere des DPMA und der Schiedsstelle nach dem Gesetz über Arbeitnehmererfindungen, letztere sind vielfach auf CD-ROM oder im Internet verfügbar). Entscheidungen der Beschwerdekammern des EPA sind mit Aktenzeichen, Fundstelle im ABl EPA und in GRUR Int, soweit dort veröffentlicht, ausnahmsweise auch mit anderen Fundstellen, zitiert, gedruckte Entscheidungen in der Regel mit einem Schlagwort; die Entscheidungen sind durchwegs im Internet abrufbar. Entscheidungen des EuGH sind mit der Fundstelle in der Amtlichen Sammlung (Slg), solange und soweit dort veröffentlicht, und einer weiteren Fundstelle zitiert. Bei ausländischen Entscheidungen richtet sich die Zitierweise in erster Linie nach der Erreichbarkeit der Fundstelle. Entscheidungsanmerkungen und -besprechungen sind am allgemeinen nicht zitiert. Nicht zitiert werden grds die Schulte-Kartei sowie nur gegen Gebühr zugängliche Datenbanken wie BeckOnline oder juris. Ob online-Kommentare zitiert werden, ist den Bearbeitern überlassen.

Die Gerichte (außer EuGH, BVerfG, BGH, BVerwG, BPatG uä) werden grundsätzlich nach ihrem Sitz genannt, auch wenn ihre amtliche Bezeichnung abweicht (zB LAG Hannover statt Niedersächsisches LAG, OVG Bautzen statt Sächsisches OVG, ehemaliger VGH Stuttgart statt Württemberg-Badischer VGH).

In diesem Kommentar wird wie bisher im Text des deutschen Patentgesetzes (anders in einigen neueren Gesetzes- und Verordnungstexten sowie nunmehr in der Schweiz) bei der Verwendung generischer (dh nicht auf eine bestimmte Person bezogener) Begriffe generell die generische Form (traditionell meist das grammatische Maskulinum) verwendet, dies nicht etwa, um Frauen zu diskriminieren (vgl hierzu etwa Ulrike Spangenberg Alltag oder Diskriminierung? KJ 2018, 345), sondern einmal, weil generische Bezeichnungen nichts über das natürliche Geschlecht der mit ihnen bezeichneten Person aussagen (sollten), zum anderen, weil sie fast immer knapper als andere sind (vgl Art 8 Abs 2 schweiz PatGG: „Dem Bundespatentgericht gehören zwei hauptamtliche Richterinnen beziehungsweise Richter sowie eine ausreichende Anzahl nebenamtlicher Richterinnen beziehungsweise Richter an. Die Mehrheit der nebenamtlichen Richterinnen beziehungsweise Richter muss technisch ausgebildet sein."), der Verlag darauf achtet, dass der Kommentar einen vorgegebenen Umfang nicht überschreitet, und eine rechtliche Differenzierung schon nach Art 3 Abs 2 Satz 1 GG grundsätzlich ausgeschlossen ist. Der Gebrauch des generischen Maskulinums bringt nach höchstrichterlicher Rechtsprechung keine Geringschätzung gegenüber Personen zum Ausdruck, deren natürliches Geschlecht nicht männlich ist (BGH, Urteil vom 13.3.2018 VI ZR 143/17, NJW 2018, 1671). Dass die Doppelverwendung der männlichen und der weiblichen („,bipolaren“) Bezeichnung nicht alle Schwierigkeiten beseitigt, belegt BVerfG vom 8.11.2017 1 BvR 2019/16, NJW 2017, 3643. Die sich damit vielleicht als nicht angemessen angesprochen ansehenden Präsidentinnen, Richterinnen, Patentanwältinnen, Protokollführerinnen, Klägerinnen, Nebenintervenientinnen usw mögen Nachsicht üben! Selbstverständlich wird bei generisch weiblichen Bezeichnungen (wie „die Partei“ oder „die Gesellschaft“) das generische Maskulinum nicht benutzt. 